NUCLEAR FUSION

Tokamak Excels

from a Correspondent

THE conference on plasma physics and controlled fusion research organized by the International Atomic Energy Agency at Madison, Wisconsin, from June 17 to 23 was the fourth in the series of meetings which are held at three-year intervals. They constitute the forum for the international exchange of information on progress towards an ultimate power-generating fusion reactor. At present the principal concern is the plasma confinement problem, and in spite of the title the only plasma physics covered was that held to be directly relevant to fusion.

Many different schemes for plasma confinement are under study and a good deal of attention is given at these international meetings to their relative progress and future promise. This latest meeting was fairly unremarkable in the sense that no outstanding new results or concepts were presented; the remarkable feature was that for two successive meetings in the series the Russian Tokamak system has maintained a lead both in performance and promise over rival schemes.

Thus Dr E. Meservey (Princeton University) presented results from the Princeton Tokamak which in broad terms confirmed those obtained earlier by Russian workers on their T-3 machine in Moscow. There are some differences and attention was naturally concentrated on these. For example, the electron temperature is strongly peaked near the axis in the American machine, whereas in the Russian one the profile is relatively flat. There are other differences which seem to be attributable to differences in interpretation of essentially the same experimental data. Thus the Russians think that the containment time for particles greatly exceeds that for the energy whereas the American view is that they are about equal.

Dr V. S. Strelkov (Kurchatov Institute, Moscow) presented results from the latest Russian Tokamak, T-4. This has a higher magnetic field $(50 \mathrm{kG})$ and the stabilizing copper shell is nearer to the plasma than in T-3. The plasma current has thus been increased by almost a factor of two; the ion temperature as deduced from the neutron yield reaches $650 \mathrm{eV}$. With a current of 110 $\mathrm{kA}$ the electron temperature is $\sim 2 \mathrm{keV}$, the plasma density is $3 \times 10^{13} \mathrm{~cm}^{-3}$ and the containment time is 10 milliseconds. These are impressive numbers but they fall short by about one order in both temperature and density and two orders in containment time over the requirements for a fusion reactor. It should be emphasized that although many features of the Tokamak discharge are not understood there are fairly clear, if expensive, lines along which the next experiments should proceed.

Whether some tortoise will eventually overtake the Tokamak hare is an interesting question. The stellarator which is closely related to the Tokamak, but much more expensive and difficult to build, seems to be undergoing a random walk rather than progress. Dr P. Reynolds (UKAEA, Culham Laboratory) presented results from his small stellarator which agree very well with the theory of classical containment. By contrast Dr K. Myamoto (Nagoya University) finds fluctuations and anomalous losses in his very similar machine. For the largest stellarator at present operating in the world, the Uragan machine at Kharkov, Dr K. N. Stepanov reported electron temperatures up to $400 \mathrm{eV}$, density $\sim 5 \times 10^{12} \mathrm{~cm}^{-3}$ and containment time $\sim 300 \mu \mathrm{s}$.

Research on high beta toroidal confinement is still in an early stage as groups previously working on linear theta pinches move into the field. Typical results on screw pinches reported from Jutphaas, Culham and Garching were electron density $\sim 10^{16} \mathrm{~cm}^{-3}$, plasma temperature $\sim 100 \mathrm{eV}$ and containment time $\sim 10 \mu \mathrm{s}$. This configuration relies for its equilibrium on toroidal current flow in the outer low density region. The rapid decay of this current is believed to be the limiting factor and Dr P. C. T. van der Laan (Institute for Plasma Physics, Jutphaas) discussed a variety of possible explanations without reaching any definite conclusion. Dr H. A. B. Bodin (UKAEA, Culham Laboratory) presented results on the diffuse pinch configuration which were encouraging in the sense that the configuration could be set up and its stability agreed roughly with theory but discouraging in that the temperature was limited by impurity radiation to $\sim 10 \mathrm{eV}$. Results from a segment of the Scyllac torus reported by Dr F. L. Ribe (Los Alamos Scientific Laboratory) showed control of the toroidal drift by the addition of $l=0$ and $l=1$ fields.

In mirror research the chief result was that reported by Dr F. H. Coensgen (Lawrence Radiation Laboratory, Livermore) who finds that plasma is lost from his $2 \mathrm{X}$ machine at about ten times the classical rate. This fast loss is accom-

\title{
Mixed RNAs in Transformed Cells
}

IN Nature New Biology next Wednesday, Wall and Darnell report a series of elegant DNA.RNA hybridization experiments which establish once and for all that sequences of SV40 RNA occur in large molecules covalently linked to sequences of host cell RNA in the nuclei of mouse cells transformed by SV40.

In 1968 Sambrook and his colleagues showed that, in stably transformed cells, SV40 DNA molecules are integrated into DNA molecules of the host genome. Since then, although the number of integrated SV40 genomes in transformed cells has remained a matter of dispute, numerous groups have found that SV40 RNA is present in both the nucleus and cytoplasm of transformed cells. Further, it is generally agreed that SV40 RNA occurs in the nucleus in molecules which are longer than a single complete SV40 genome and in the cytoplasm the SV40 RNAs associated with polysomes are no longer than a single SV40 genome. There is every reason to believe that these polysomal SV40 RNAs are derived by cleavage from the large nuclear RNA molecules which contain SV40 sequences, but how do these large nuclear RNAs themselves arise?

There are two obvious possibilities which have been canvassed. If several SV40 genomes are integrated in tandem in each transformed cell they might be transcribed as a single unit to yield RNA molecules longer than a single SV40 genome and containing nothing but SV40 sequences. Alternatively, the SV40 genomes may be integrated singly in such a way that they are always transcribed together with some of the adjacent host DNA; in which case the size of these RNAs would depend on how much of the host genome is transcribed and they should contain host sequences either at the $5^{\prime}$ or $3^{\prime}$ or both sides of the SV40 sequences.

To decide this issue, Wall and Darnell first hybridized to SV40 DNA RNAs from the nucleus and cytoplasm of transformed and untransformed cells. They then recovered the hybridizing RNA from the hybrids, which must contain SV40 sequences, and tested to see if it would also hybridize with cellular DNA from untransformed cells. They detected nuclear RNA molecules from transformed cells which hybridized with both SV40 and cellular DNAs. Wall and Darnell conclude therefore that in transferred cells at least some SV40 DNA molecules are transcribed together with some host DNA to give large and mixed RNA molecules. And although their results do not prove the idea that these mixed molecules are precursors of the small SV40 RNAs associated with polysomes they do not contradict it. By further refining the hybridization techniques and ultimately by fingerprinting these various RNAs it should be possible to decide if they are related as precursors and products and to learn something of the mechanisms which control transcription and messenger RNA maturation in eukaryotes. 
panied by evidence of instabilities with frequencies near the ion cyclotron frequency. Because a mirror reactor requires near-classical confinement this result is not encouraging; it is possible, however, that the necessary quiescence can be achieved by careful machine design and control of the particle velocity distribution.

Theoretical work presented at the conference dealt chiefly with plasma confinement and stability in the Tokamak. There is now a fair measure of agreement on the classical theory of confinement in symmetric low beta systems. Attention is now paid to the effect of residual fiuctuations on such systems and contributions from Dr S. Yoshikawa (Princeton University) and Drs A. A. Galeev and R. Z. Sagdeev (Kurchatov Institute, Moscow) reached similar conclusions by rather different routes. This work emphasizes the role of the poloidal magnetic field in plasma confinement, the much stronger toroidal field performing only an auxiliary function. On the question of stability, the situation is not clear ; a contribution by Drs D. Pfirsch and H. Tasso (Institute for Plasma Physics, Garching) which showed the Tokamak to be unstable against a simple mode will undoubtedly be the subject of much critical attention.

For the first time at these meetings a session was devoted to reactor systems. The broad conclusion from this session was that within the necessarily very wide margins of error, fusion as a power source might be competitive with other systems and might offer substantial environmental advantages.

\section{RNA}

\section{Messenger's Burden}

from our Molecular Biology Correspondent ONE of the strangest molecules that explorers of the eukaryotic nucleus have brought to light is polyadenylic acid, in almost monodisperse form. This observation became more than a mere unexplained curiosity when Lim and Canellakis reported last year that haemoglobin messenger RNA contains a long tract of poly $\mathrm{A}$, which apparently largely accounts for the discrepancy between its observed and expected molecular weight.

The question of how general this phenomenon may be has now been taken up in several laboratories, and a clutch of three articles on the subject is to be found in the current issue of the Proceedings of the US National Academy of Sciences.

Lee, Mendecki and Brawerman (68, 1331 ; 1971) have examined the messenger RNA of ascites cells. They find that at $p \mathrm{H} \mathrm{9,} \mathrm{though} \mathrm{not} \mathrm{under}$ more nearly neutral conditions, a large proportion of the rapidly labelling
RNA fraction of polysomes is specifically trapped on a membrane filter. The fraction so obtained leaves after digestion with pancreatic or $T_{1}$ ribonuclease a sedimentable core consisting largely or wholly of adenylic acid residues, a label in adenosine being almost completely recovered in this resistant fragment, whereas a label in uridine vanishes. Moreover, the behaviour of synthetic poly $A$ on the membrane filters makes it clear that it is the poly $\mathbf{A}$ segment that causes the messenger to be retained. The poly A core recovered from the messenger sediments at about the same rate as tRNA. Another property of the messenger, conferred on it by its poly A, is its tendency, depending on the salt conditions, to enter the wrong phase during phenol extraction.

Very similar results were obtained by Edmonds, Vaughan and Nakazato (ibid., 1336) for the messenger RNA of HeLa cells. The poly A core was shown by polyacrylamide gel electrophoresis to have a very narrow molecular weight distribution, and an esti- mated length of 150-200 nucleotides. This is in fact rather too high to be accounted for by the estimated poly $A$ content of the messenger, but it does not necessarily follow that some of the messenger is devoid of poly $\mathbf{A}$ : a number of other explanations are possible, for example that some of the label goes into other kinds of RNA than messenger.

The chief object of the article, however, is to treat the poly $A$ as an identifying feature, and to look for its presence in the rapidly labelling polydisperse high molecular weight RNA present in the nucleus, which many workers in the field would like to believe is a messenger precursor. Poly $A$ is indeed recovered from this material by digestion, and is of the same size as that found in the messenger. It makes up only some 0.5 per cent of the total. That the poly $\mathrm{A}$ is a part of a large RNA molecule was demonstrated by its failure to dissociate and redistribute itself between labelled and unlabelled nuclear RNA after addition of a high concentration of an organic

\section{Acceleration of Cosmic Rays}

THE drawback of the venerable Fermi mechanism for the generation of the cosmic ray spectrum is the large energies that the particles need to have before the mechanism comes into play. Although the scattering of cosmic ray particles by irregularities in the magnetic fields of interstellar space will produce an energy distribution which matches the experimental data on cosmic rays, as Fermi suggested the particles have to have initial energies of 200 $\mathrm{MeV}$ if they are protons, and higher energies if they are heavier particles.

A process which will raise the energies of particles from thermal energies to sufficiently high energies for injection into the Fermi mechanism is suggested in next Monday's number of Nature Physical Science. A group at the University of Bologna argue that particles able to take part in the Fermi mechanism may come from the nucleus of the galaxy, where they have been accelerated from thermal energies by interactions with the magnetic fields of neutron stars.

This notion seems to be inspired by the pulses recorded by Weber's gravitational wave equipment, and whatever the explanation of the data published by Weber, his results have stimulated theoretical work on the conditions in the nuclei of galaxies. Interactions among clusters of collapsed objects at the centre of the galaxy might be a source of gravitational waves, but there are other more prosaic reasons for supposing that the nuclei of galaxies contains dense matter. In any case, the
Bologna group consider a cluster of neutron stars, a proportion of which have strong magnetic fields, and show how charged particles can gain energy by being scattered by the fields.

Protons need to be scattered only twice to gain the $200 \mathrm{MeV}$ that is the entry ticket for the Fermi process. Heavier particles may need to be scattered more than twice, but there is no reason why the $1 \mathrm{BeV}, 20 \mathrm{BeV}$, and $300 \mathrm{BeV}$ that are the required injection energies for alpha particles, oxygen, and iron respectively should not be achieved in this way.

Little more can be said without knowing more about the abundances of the elements in the interstellar gas in the galactic nucleus, and little light is likely to be thrown on this aspect of the problem for many years. The hope is, of course, that the abundances, together with the fact that it becomes progressively more difficult to provide the heavier elements with the necessary injection energy, will provide a match with the element abundances observed from measurements on cosmic rays.

The Bologna article is well in tune with recent trends in astrophysics. The beginnings of a realization of the parts played by high energy processes and by collapsed objects are suggesting several ways in which the acceleration of cosmic rays may be accomplished. For example, particles thrown out of the atmospheres of pulsars by their rapid rotation rates may also have high enough energies to take part in cosmic ray processes. 